\title{
Análisis comparativo de la competitividad y productividad de la empresa Hukk Makilla, basada en la metodología del Mapa de Competitividad Promperú, 2014
}

\author{
Comparative analysis of the competitiveness and productivity of \\ Hukk Makilla company, based on the methodology of Promperú \\ Competitiveness Map, 2014
}

Víctor Quintana Palacios' , Jean Aliaga Huaynalaya ${ }^{2}$, Magda Conovilca Camasca ${ }^{3}$, Denis Tapia Rodríguez ${ }^{4}$ Universidad Continental

\section{RESUMEN}

Objetivos: Describir el nivel de competitividad y productividad de la empresa Hukk Makilla del sector textil del distrito de Huayucachi, Huancayo. Métodos: Fue una investigación aplicada, de diseño preexperimental. La recolección de datos se realizó a través de la aplicación del mapa de competitividad empresarial, que permitió obtener información del nivel de competitividad y productividad de la empresa. La muestra fue la empresa textil Hukk Makilla que participó en el Programa de Training en Gestión Exportadora promovida por Promperú. Las mediciones, siguiendo el diseño, fueron al inicio y al final, para luego determinar los respectivos diagnósticos de competitividad de la empresa. Resultados: La empresa aumentó la competitividad en un $28 \%$, de $45 \%$ a $73 \%$. El crecimiento general de competitividad por áreas fue de $83 \%$ en planeamiento estratégico, $79 \%$ en contabilidad $y$ finanzas, $77 \%$ en sistemas de información, 74 $\%$ en gestión ambiental, $73 \%$ en producción y operaciones, $69 \%$ en comercialización, $65 \%$ en recursos humanos y $62 \%$ en aseguramiento de la calidad. Conclusiones: La gestión empresarial permite a las empresas aumentar su competitividad y productividad como en este caso al implementarse medidas innovadoras en diversas áreas de la empresa ha permitido obtener mejoras.

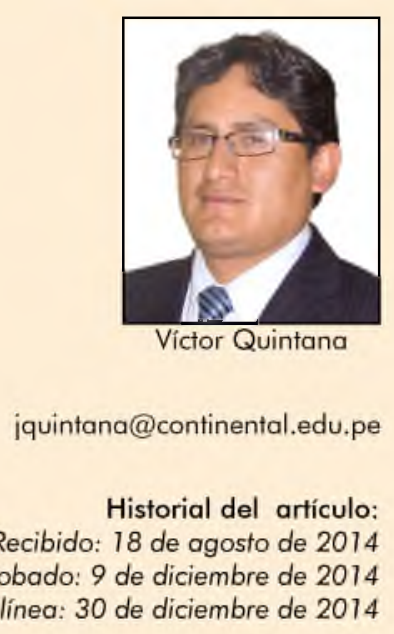

Palabras clave: Empresa, mapa de competitividad, gestión empresarial, sector textil.

1 Magíster en Administración de Negocios, docente de la Universidad Continental.

2 Estudiante de Economía de la Universidad Continental.

3 Estudiante de Contabilidad de la Universidad Continental.

4 Economista, docente de la Escuela de Economía de la Universidad Continental. 


\section{ABSTRACT}

Objectives: To describe the level of competitiveness and productivity of Hukk Makila's company in the textile sector, situated in the district of Huayucachi, Huancayo. Methods: This is an applied research of pre- experimental design. The data collection was carried out through the application map of business competitiveness which facilitated the researcher to gather information on the level of competitiveness and productivity of the company. The purposive sampling was the company Hukk Makilla which participated in the Export Managing Program Training sponsored by Promperu. The measurements, following the design, were made in the initial and finale, in order to determine the respective diagnoses of Hukk Makila's competitiveness. Results: The organization increased its competitiveness by $28 \%$, from $45 \%$ to $73 \%$. The growth of competitiveness by areas was $83 \%$ in Strategic Planning, $79 \%$ in Accounting and Finance, $77 \%$ Information Systems, 74 \% in environmental management, $73 \%$ in Production and Operations, $69 \%$ Marketing, $65 \%$ in Human Resources, and $62 \%$ on Quality Assurance. Conclusions: It was concluded that the Business Management enables companies to increase their competitiveness and productivity as it has shown in this case applying innovative measures in different areas of the enterprise to get better improvements.

Keywords: Enterprise, competitiveness map, business management, textile sector.

\section{INTRODUCCIÓN}

En los últimos años, ha surgido gran cantidad de estrategias para el mejoramiento de los procesos productivos, comerciales y de gestión, llegando hasta las ventajas competitivas. La competitividad es un aspecto que ha adquirido mayor relevancia en el campo empresarial y de las Mypes aun en la región Junín.

Según Padilla la competitividad está relacionada con la capacidad de incrementar el nivel de vida de los habitantes, de generar incrementos sostenidos en productividad, de insertarse exitosamente en los mercados internacionales, entre otros (1).

Dussel la define como el proceso de integración dinámica de países y productos a mercados internacionales, dependiendo tanto de las condiciones de oferta como de las de demanda (2).

De acuerdo con Abdel y Romo, existe un conjunto de estudios iniciales sobre la competitividad que estuvieron enfocados al análisis del débil desempeño de las empresas estadounidenses con respecto a las de otros países, en especial Japón, en sectores dominados tradicionalmente por los norteamericanos (3).

Actualmente Promperú establece en nuestro país los mapas de competitividad, los que son definidos por esta institución como una herramienta que nos ayuda a medir el nivel de competitividad de las empresas, mediante entrevistas formuladas en todas las áreas relacionadas con la empresa, desde el planeamiento estratégico, seguido de la producción y operaciones, el aseguramiento de la calidad, la comercialización, la contabilidad, los recursos humanos, la gestión ambiental, hasta los sistemas de información, considerándose en cada área los aspectos más importantes (4).

La gestión empresarial abarca un conjunto detécnicas y herramientas que se aplican a la administración de una empresa. Su objetivo fundamental es mejorar la productividad, sostenibilidad y competitividad, asegurando la viabilidad de la empresa en el largo plazo.

El objetivo de este estudio fue determinar la relación existente entre la gestión empresarial y el mapa de competitividad y productividad de la empresa Hukk Makilla del distrito de Huayucachi. 


\section{MATERIAL Y MÉTODOS}

La investigación fue aplicada, mediante el estudio de caso, de diseño preexperimental, de tipo transversal. La recolección de datos fue a través de la aplicación de un instrumento de tipo cuestionario con preguntas cerradas y adaptado del mapa de competitividad empresarial, que permitió recoger información del nivel de competitividad y productividad de la empresa.

El estudio se realizó en la empresa Hukk Makilla S. A. C. de acuerdo al diseño se aplicaron dos mediciones, inicial y final, para luego determinar los respectivos diagnósticos de competitividad de la empresa.

Los procedimientos utilizados iniciaron con un diagnóstico situacional de la competitividad y productividad, para luego aplicar y utilizar técnicas de gestión empresarial como planeamiento estratégico, plan de marketing, plan de ventas, plan de las $5 \mathrm{~S}$, programación de producción, control de inventarios, diseño de presupuestos, análisis de costos estratégicos, diseño y elaboración de sistemas de información y control, manuales de funciones y procedimientos y políticas de control interno, enfocadas a mejorar la competitividad y productividad en dicha empresa a través de la realización de un diagnóstico previo y posterior.

\section{RESULTADOS}

De acuerdo con el diagnóstico comparativo inicial y final, establecido tras nueve meses de gestión empresarial, el crecimiento diferencial de la competitividad alcanzado en este período por la empresa Hukk Makilla fue de $28 \%$ ( 1,4 puntos $)$, de 45 $\%$ a $73 \%$, es decir, de 2,24 a 3,64 puntos (tabla $N^{\circ} 1$ ).

Según el diagnóstico inicial de competitividad por áreas (tabla $\mathrm{N}^{\circ} 2$ y figura $\mathrm{N}^{\circ} 1$ ), la empresa obtuvo un promedio de $45 \%$ y ninguna de sus áreas superó el $60 \%$, lo
Tabla $N^{\circ}$ 1: Crecimiento diferencial promedio de competitividad.

\begin{tabular}{lccr}
\hline & $\begin{array}{c}\text { Diagnóstico } \\
\text { inicial de } \\
\text { competitividad }\end{array}$ & $\begin{array}{c}\text { Diagnóstico } \\
\text { final de } \\
\text { competitividad }\end{array}$ & Avance \\
\hline Puntaje & 2,24 & 3,64 & 1,4 \\
Porcentaje & $45 \%$ & $73 \%$ & $28 \%$ \\
\hline
\end{tabular}

Tabla $N^{\circ}$ 2: Diagnóstico inicial de la competitividad por áreas.

\begin{tabular}{|c|c|c|}
\hline \multicolumn{3}{|c|}{ Diagnóstico integral de competitividad } \\
\hline 1. Planeamiento estratégico & 1,95 & $39 \%$ \\
\hline 2. Producción y operaciones & 2,36 & $47 \%$ \\
\hline 3. Aseguramiento de la calidad & 2,20 & $44 \%$ \\
\hline 4. Comercialización & 2,14 & $43 \%$ \\
\hline 5. Contabilidad y finanzas & 2,59 & $52 \%$ \\
\hline 6. Recursos humanos & 2,53 & $51 \%$ \\
\hline 7. Gestión ambiental & 2,30 & $46 \%$ \\
\hline 8. Sistemas de información & 1,36 & $27 \%$ \\
\hline Promedio de la empresa & 2,24 & $45 \%$ \\
\hline
\end{tabular}

que denota la existencia de puntos débiles en el desarrollo de la gestión empresarial y obliga a implementar los cambios.

Examinadas cada una de las áreas, el diagnóstico inicial fue el siguiente:

- En planeamiento estratégico: existía un planeamiento escrito pero con problemas en la implementación.

- En producción y operaciones: no contaba con un flujograma de producción que evalue el tiempo de producción por producto. Además, no se ha determinado la capacidad instalada y productiva. En el ámbito de operaciones, la empresa no contaba con diseños y programas de producción para incrementar la producción y comercialización de nuevos diseños sobre la base de una respuesta del estudio de mercado a fin de obtener la máxima rentabilidad.

- En aseguramiento de la calidad: realizaba un control de cada proceso productivo, pero no contaba con una estructura de control documentada que se aplica.

- En comercialización: no contaba con un 


\begin{tabular}{|c|c|c|}
\hline \multirow[b]{2}{*}{ 1. Planeamiento estratégico } & \multicolumn{2}{|c|}{$\begin{array}{c}\text { Calificación de } \\
\text { la empresa }\end{array}$} \\
\hline & 4,13 & $83 \%$ \\
\hline 2. Producción y operaciones & 3,65 & $73 \%$ \\
\hline 3. Aseguramiento de la calidad & 3,11 & $62 \%$ \\
\hline 4. Comercialización & 3,46 & $69 \%$ \\
\hline 5. Contabilidad y finanzas & 3,95 & $79 \%$ \\
\hline 6. Recursos humanos & 3,23 & $65 \%$ \\
\hline 7. Gestión ambiental & 3,69 & $74 \%$ \\
\hline 8. Sistemas de información & 3,84 & $77 \%$ \\
\hline Promedio de la empresa & 3,64 & $73 \%$ \\
\hline \multicolumn{3}{|c|}{$\begin{aligned} & \text { Tabla } N^{\circ} 4: \begin{array}{l}\text { Crecimiento diferencial } \\
\text { competitividad por áreas. }\end{array} \\
&\end{aligned}$} \\
\hline \multirow[b]{2}{*}{ Áreas diferencial } & \multicolumn{2}{|c|}{$\begin{array}{l}\text { Calificación de } \\
\text { la empresa }\end{array}$} \\
\hline & Inicial & Final \\
\hline 1. Planeamiento estratégico, $44 \%$ & $39 \%$ & $83 \%$ \\
\hline 2. Producción y operaciones, $26 \%$ & $47 \%$ & $73 \%$ \\
\hline 3. Aseguramiento de la calidad, $18 \%$ & $\% 44 \%$ & $62 \%$ \\
\hline 4. Comercialización, $26 \%$ & $43 \%$ & $69 \%$ \\
\hline 5. Contabilidad y finanzas, $27 \%$ & $52 \%$ & $79 \%$ \\
\hline 6. Recursos humanos, $14 \%$ & $51 \%$ & $65 \%$ \\
\hline 7. Gestión ambiental, $28 \%$ & $46 \%$ & $74 \%$ \\
\hline 8. Sistemas de información, $50 \%$ & $27 \%$ & $77 \%$ \\
\hline Promedio de la empresa, $28 \%$ & $45 \%$ & $73 \%$ \\
\hline
\end{tabular}

catálogo de productos y clientes, sus ventas eran limitadas a las visitas de turistas.

- En contabilidad y finanzas: no tenia un sistema de control interno, estado de pérdidas y ganancias proyectado, flujo de caja, ni un sistema de costos por producto.

- En recursos humanos: presentaba limitaciones para indicar las sanciones y beneficios al personal donde se considere la productividad y las funciones de cada área de la empresa.

- En gestión ambiental: no contaba con la documentación requerida sobre desempeño ambiental o cuidado del medio ambiente que promueva. Solo de forma práctica desarrolla el proceso de cuidado medioambiental.

- En sistemas de información: no tenía sistemas de información que le permita manejar datos como montos de ventas de acuerdo con los pedidos y pueda dar un informe en la fecha solicitada, calculo de costos reales por productos y tipo de material, entre otros instrumentos que puedan sintetizar la información.

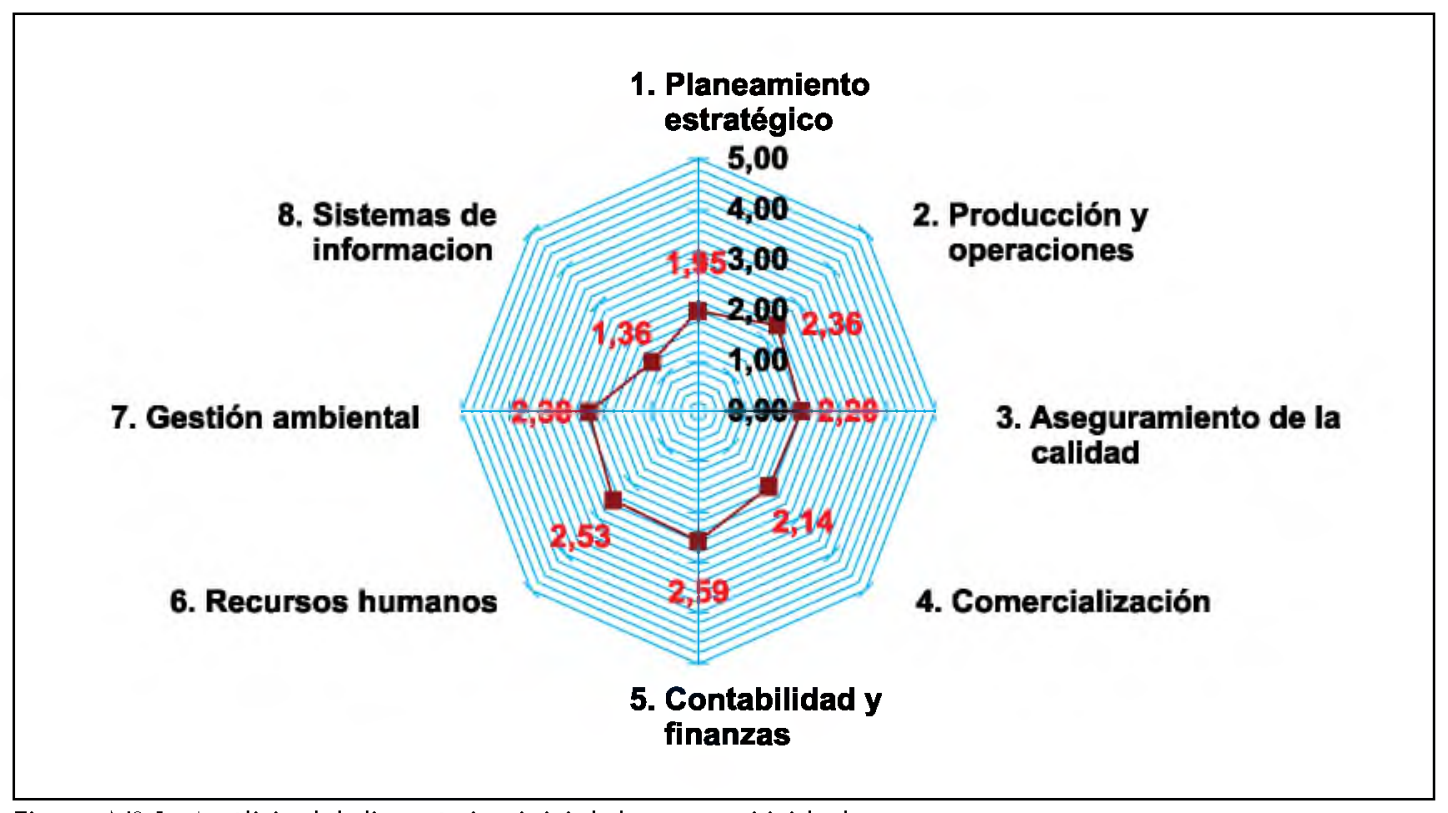

Figura $\mathrm{N}^{\circ}$ 1: Análisis del diagnóstico inicial de competitividad. 
Tabla $N^{\circ}$ 5: Acciones implementadas en cada área para el crecimiento de la competitividad de la empresa Hukk Makilla.

\begin{tabular}{|c|c|}
\hline Planeamiento Estratégico & $\begin{array}{l}\text { - Elaboraron la visión, misión, objetivos, metas, acciones e indicadores. } \\
\text { - Elaboración de una matriz FODA. } \\
\text { - Plantearon sus estrategias para cumplir sus objetivos. } \\
\text { - Planificación del presupuesto trimestral. }\end{array}$ \\
\hline Producción y Operaciones & $\begin{array}{l}\text { - Esquema del control de los procesos productivos. } \\
\text { - Medición de la capacidad productiva de la empresa. } \\
\text { - Programa de mantenimiento de la maquinaria. } \\
\text { - Delimitación y señalización de las áreas. } \\
\text { - Elaboración de una ficha para control de ingreso y salida de materia prima } \\
\text { (las tejedoras trabajan en sus casas). } \\
\text { - Elaboración de una colección para mujeres con temas sobre el nevado } \\
\text { Huaytapallana. }\end{array}$ \\
\hline Aseguramiento de la calidad & $\begin{array}{l}\text { - Plantearon un esquema de control de calidad indicando en cada proceso las } \\
\text { inspecciones que se deben hacer. } \\
\text { - Elaboración de una tabla de tallas y fichas técnicas para todo los productos. }\end{array}$ \\
\hline Comercialización & $\begin{array}{l}\text { - Segmentación del publico. } \\
\text { - Plantearon un plan para el desarrollo de marca. }\end{array}$ \\
\hline Contabilidad y finanzas & $\begin{array}{l}\text { - Elaboración de una estructura de costos. } \\
\text { - Elaboración de balance general proyectado. } \\
\text { - Elaboración de estados de resultados proyectados. } \\
\text { - Elaboración de libro diario. } \\
\text { - Establecer la política de las cuentas por cobrar y pagar. }\end{array}$ \\
\hline Recursos humanos & $\begin{array}{l}\text { - Actualización del organigrama de la empresa. } \\
\text { - Plantearon las políticas de seguridad y salud ocupacional. } \\
\text { - Plan de actividades con el personal a fin de logra la identificación con la } \\
\text { empresa. }\end{array}$ \\
\hline Gestión ambiental & $\begin{array}{l}\text { - Elaboración de un plan de desechos. } \\
\text { - Realización de manualidades con el reciclaje de la lana. }\end{array}$ \\
\hline Sistemas de información & $\begin{array}{l}\text { - Sistema de control de inventario } \\
\text { - Sistema de control de ventas y generación de reportes }\end{array}$ \\
\hline
\end{tabular}

El crecimiento general de competitividad por áreas fue de $83 \%$ en planeamiento estratégico (superior a los 4 puntos), $79 \%$ en contabilidad y finanzas, $77 \%$ en sistemas de información, $74 \%$ en gestión ambiental, $73 \%$ en producción y operaciones, $69 \%$ en comercialización, $65 \%$ en recursos humanos y $62 \%$ en aseguramiento de la calidad (tabla $\mathrm{N}^{\circ} 3$ ).

El crecimiento diferencial por áreas, fue mayor en sistemas de información, $50 \%$ adicional, de $27 \%$ a $77 \%$; planeamiento estratégico $44 \%$, gestión ambiental

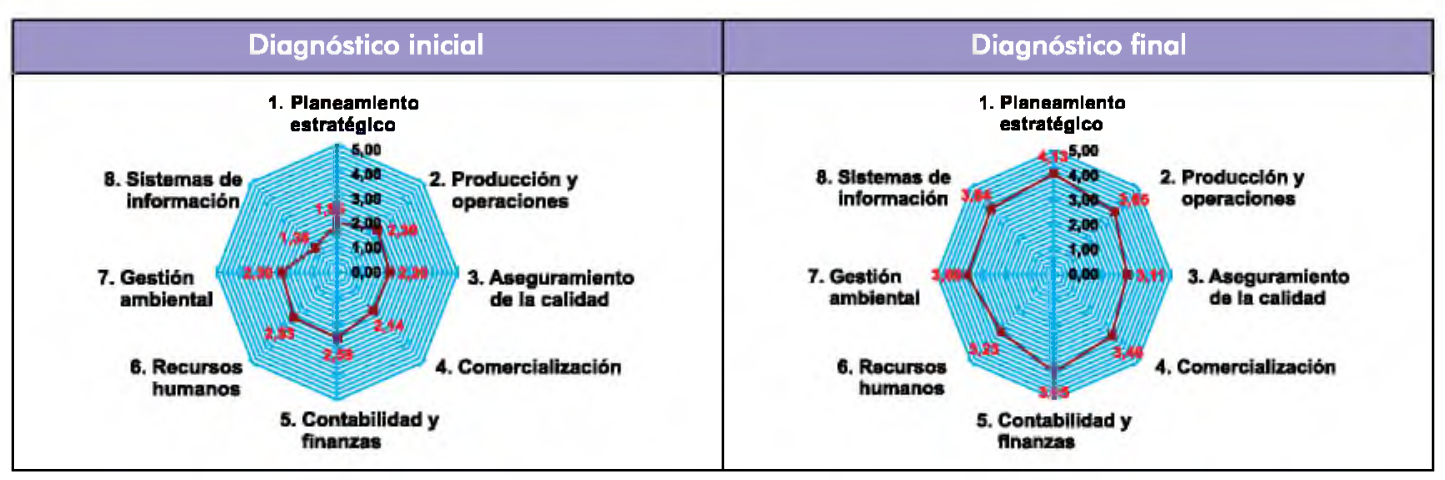

Figura $\mathrm{N}^{\circ}$ 2: Resultado comparativo de competitividad. 
$28 \%$, contabilidad y finanzas $27 \%$, comercialización $26 \%$, producción y operaciones $26 \%$, aseguramiento de la calidad $18 \%$, y recursos humanos $14 \%$, con una diferencia promedio de $28 \%$, de $45 \%$ a $73 \%$ (tabla $N^{\circ} 4$ y figura $N^{\circ} 2$ ).

El crecimiento de la competitividad de la empresa fue producto de una serie de acciones implementadas durante el estudio en cada una de sus áreas (tabla $\mathrm{N}^{\circ} 5$ ).

\section{DISCUSIÓN}

La empresa logró aumentar la competitividad en $28 \%$ de $45 \%$ a $73 \%$, de acuerdo a otros estudios realizados con otras empresas en la ciudad de Huancayo también del sector textil se ha demostrado que participando bajo este programa se ha logrado los mismos resultados tal como es reportado por Loayza y otros (5).

Es probable que la aplicación de esta metodología en empresas de otros sectores industriales permita obtener mejoras en sus niveles de competitividad.

El estudio demuestra que la gestión empresarial permite a las empresas aumentar su competitividad y productividad.

\section{Agradecimientos}

A la empresa Hukk Makilla y a PromPerú por el apoyo en la investigación.

\section{REFERENCIAS BIBLIOGRÁFICAS}

1. Padilla R. Instrumento de medición de la competitividad. México: Cepal; 2006.

2. Dussel E. Un análisis de la competitividad de las exportaciones de prendas de vestir de Centroamérica utilizando los programas y la metodología CAN y MAGIC. México: Cepal; 2001.

3. Abdel G, Romo D. Sobre el concepto de competitividad. Documentos de trabajo en estudios de competitividad. México:
Instituto Tecnológico Autónomo de México; 2004.

4. Promperú [Internet]. Lima: Test de Competitividad; 2012 [Citado el 20 de febrero de 2014]. Disponible en: http:// media.peru.info/siicex/resources/ rutaexportadora/08-RE-TEST-DECOMPETITIVIDAD.pdf

5. Loayza G. Gestión empresarial, nivel de competitividad y productividad de empresas del sector textil de Huancayo. Apunt. cienc. soc. 2014; 4(1): 15-24. 